\title{
Bubbling the diagnosis: Incidental finding of non-bacterial thrombotic endocarditis in a patient with pancreatic carcinoma
}

\author{
Johannes Wild ${ }^{1}$, Simon Distelmeier ${ }^{2}$, Philipp Keil ${ }^{3}$, Arndt Weinmann ${ }^{3}$, Thomas Münzel ${ }^{1}$, \\ Julia Weinmann-Menke ${ }^{3}$, and Daniel Kraus ${ }^{3}$ \\ ${ }^{1}$ University Medical Center Mainz \\ ${ }^{2}$ University Medical Center Mainz \\ ${ }^{3}$ University Medical Center Mainz Department of Internal Medicine 1
}

March 2, 2021

\begin{abstract}
Nonbacterial thrombotic endocarditis (NBTE) is a rare condition which most often accompanies a malignant disease and involves a hypercoagulable state. We report the incidental finding of a scarce case of NBTE affecting the tricuspid valve in a patient with metastatic pancreatic carcinoma complicated by severe venous and arterial thromboembolisms.
\end{abstract}

\section{Introduction}

Diseases of the mitral and tricuspid valve are more and more recognized as a significant cause of morbidity and mortality and are in the scope of new treatment strategies for structural heart interventions ${ }^{1,2}$. Despite the triumphal march of cardiac CT and MRT, echocardiography is still the most widely available first-line imaging modality for the heart. We present a case of the incidental finding of nonbacterial thrombotic endocarditis of the tricuspid valve in a patient with metastatic pancreatic carcinoma detected by point-ofcare echocardiography on a non-cardiological ward.

\section{Case report}

A 67-year-old female patient with metastatic pancreatic carcinoma was admitted to the department of gastrooncology in our university medical center for the initiation of palliative chemotherapy. The carcinoma was first detected by CT and diagnosed by confirmatory histology using ultrasound guided biopsy two weeks prior to the admission. In addition to the primary tumor, CT-scans had detected multiple liver metastases (Figure 1), so palliative chemotherapy with FOLFIRINOX (oxaliplatin, irinotecan, leucovorin and fluorouracil) was recommended. Initially, the therapy was planed in an outpatient setting, but due to dyspnea and progressive thrombocytopenia, the patient was admitted to the hospital.

On admission, the patient reported worsening dyspnea for about two weeks which now met the criteria of NYHA stage III. Furthermore, she reported that a deep vein thrombosis of the left lower leg had been diagnosed 10 days before admission. Anticoagulant treatment with apixaban $5 \mathrm{mg}$ twice daily had been initiated, but recently discontinued by her family physician due to severe thrombocytopenia of so far unknown origin. The patient had no history of cardiovascular disease. During physical exam, we did not find typical clinical sings of heart failure or deep vein thrombosis but petechiae on different sites of the skin on both lower legs.

Laboratory findings confirmed severe thrombocytopenia and highly elevated D-dimer levels reflecting a hypercoagulatory state. Given the severe shortness of breath and the deep vein thrombosis, an immediate CT-angiogramm of the chest was planned to rule out pulmonary embolism. 
In order to apply intravenous contrast medium and after multiple attempts at inserting a peripheral venous catheter had failed, a central venous catheter was inserted into the right internal jugular vein. Following insertion, bedside ultrasound was used for confirmation of the central venous placement by visualizing bubble artifacts in the right atrium after injection of agitated saline through the distal port ${ }^{3}$.

Unexpectedly, during visualization of the right atrium and right ventricle in subcostal view, transthoracic echocardiography (using a Philips Affinity 70 ultrasound system) revealed large masses adherent to the tricuspid valve leaflets (Figure $2 \mathrm{~A} / \mathrm{B}$ ). The differential diagnosis included thrombi, bacterial vegetations and metastases. A CT was obtained immediately and demonstrated a hypodense lesion of $28 \times 20 \mathrm{~mm}$ with predominantly intra-ventricular and only a small intra-atrial proportion (Figure 3). The lesion was irregularly configured and showed multiple stripy foothills to the ventricle walls. Furthermore, bilateral segmental and subsegmental pulmonary embolisms were detected.

After an interdisciplinary discussion of the echocardiography and CT results, we interpreted the tricuspid mass as most consistent with non-bacterial thrombotic endocarditis. We explained the findings to the patient and outlined the risks and benefits of initiating the planned chemotherapy. Specifically, we were concerned that chemotherapy might destabilize the intracardial mass, causing massive pulmonary embolism On the other hand, anticoagulative therapy was deemed riskful because of the marked thrombocytopenia. Finally, not acting at all would allow the tumor to grow in an uncontrolled manner. The patient opted for the chemotherapy which was initiated the next day. During the treatment, several sets of blood cultures as well as serum procalcitonin were negative indicating no hint for infectious endocarditis. Therapeutic anticoagulation was not possible due to persistent severe thrombocytopenia and spontaneous intracutaneous and catheter-related bleeding.

Several days later, the patient developed arterial thromboses in both lower extremities with critical ischemia, reflecting an overall deranged coagulation with parallel hypercoagulation and bleeding disorder consistent with disseminated intravascular coagulation. We performed a short time echocardiographic control after the first course of chemotherapy.

Unfortunately, the masses covering the tricuspid valve leaflets increased with signs of valve dysfunction and severe tricuspid insufficiency (Figure 4). Despite severe thrombozytopenia, continuous heparin and alprostadil were started. Within a few days, the thrombocyte count reached normal levels, and serum D dimer levels declined. Naturally, toe necroses did not resolve. The patient was discharged on low molecularweight heparin in order to continue palliative chemotherapy in the outpatient department.

\section{Discussion}

Nonbacterial thrombotic endocarditis (NBTE, formerly known as marantic endocarditis) has first been described by Ziegler in 1888 , as fibrin thrombi on normal or degenerated cardiac valves ${ }^{4}$. Postmortem, the histological diagnosis of NBTE is defined by the presence of a mixture of platelets and fibrin on the valvular leaflet without detecting micro-organisms destructing the valve. In clinical routine, NBTE is rarely diagnosed antemortem and is likely to be underdiagnosed and overlooked ${ }^{5}$ as a definitive histological diagnosis is not possible in most cases. In the presented case, a histological confirmation was also not feasible as it would have endangered the patient without affecting the further management. So, the diagnosis was based on appropriate imaging (echocardiography and $\mathrm{CT}$ ) combined with the clinical findings. We resigned from additional MRI imaging as it would not have changed the clinical management of the patient.

A large autopsy study from 1976 reports an incidence of $1.6 \%$ of NBTE in the adult autopsy population ${ }^{6}$. Coagulation abnormalities suggestive of disseminated intravascular coagulation were present in $18.5 \%$ of the cases $^{6}$. Other autopsy studies described the significantly higher prevalence of NBTE in patients with malignancies than in patients without cancer ${ }^{7,8}$. The condition is predominantly seen in patients suffering from pancreatic cancer in comparison with other carcinomas. As exemplified by our case, NBTE is significantly more common with more than $10 \%$ of all pancreatic cancer patients presenting histological features of the disease in autopsy studies $^{7}$. 
Regarding the anatomical site, the aortic valve is most often affected, followed by the mitral valve and a combination of both the aortic and mitral valves. Affections of the tricuspid or pulmonary valve are very rare reflecting only $3.6 \%$ (tricuspid valve) or $0.9 \%$ (pulmonary valve) of all cases ${ }^{9}$. When the tricuspid valve is affected, the vegetations are typically present on the atrial surface and occuring at the coapting edge of the leaflets but without altering valve function ${ }^{9}$. In the first echocardiography, our patient did not suffer from severe tricuspid insufficiency, but unfortunately in a noticeably short span of time, the regurgitation increased, indicating functional impairment of the valve by the growing mass.

Systemic embolization is known to be the main cause of morbidity in NBTE patients, not only affecting the heart valves but also peripheral intravascular thromboses. Arterial thrombosis with infarction have been described in many peripheral organs, whereas spleen and kidneys were most frequent. ${ }^{6}$ Our patient suffered from venous thromboembolism (deep vein thrombosis and subsequent pulmonary embolism) in the early stage of the disease and additionally developed peripheral arterial thrombosis most probably driven by the coagulopathy.

\section{Conclusion}

We present a rare case of severe nonbacterial thrombotic endocarditis affecting the tricuspid valve. Unfortunately, the patient developed severe tricuspid insufficiency in a noticeably short period despite ongoing non-surgical treatment. We want to highlight the unusual and incidental detection of the NTBE in our case by bedside ultrasound on a non-cardiological ward. Our case might emphasize that basic echocardiography even when done in a bedside, point-of care setting without following a formal protocol, can provide insights that help to improve patient management.

\section{Figure Legends}

\section{Figure 1}

Computed tomography (CT) scan of the abdomen demonstrating excessive hepatic metastasis (arrows for examples) of the pancreas carcinoma.

\section{Figure 2}

(A) Bedside two-dimensional transthoracic echocardiography. Parasternal short-axis view showing a large masse adhering to the tricuspid valve. $\mathrm{TV}=$ tricuspid valve, $\mathrm{RA}=\mathrm{Right}$ atrium, $\mathrm{RV}=$ Right ventricle, $\mathrm{LA}=$ Left atrium.

(B) Color doppler echocardiography reflecting tricuspid insufficiency which was estimated as non-severe by the given limited quality of the imaging in the point-of care setting.

\section{Figure 3}

Cardiac CT scan demonstrating a 28 x $20 \mathrm{~mm}$ measuring hypodense lesion with predominantly intraventricular and a small intra-atrial proportion. $\mathrm{TV}=$ tricuspid valve, $\mathrm{RA}=\mathrm{Right}$ atrium, $\mathrm{RV}=\mathrm{Right}$ ventricle, $\mathrm{LA}=$ Left atrium, $\mathrm{LV}=$ Left ventricle, $\mathrm{PA}=$ Pulmonary artery.

\section{Figure 4}

(A) Two-dimensional transthoracic echocardiography,(B) X-plane imaging and (C) color doppler echocardiography. Optimized apical four chamber view to demonstrate the NTBE of the tricuspid valve. TV = tricuspid valve, $\mathrm{RA}=$ Right atrium, $\mathrm{RV}=$ Right ventricle, $\mathrm{LA}=$ Left atrium, $\mathrm{LV}=$ Left ventricle.

\section{Author contributions}

JW: Imaging, concept and drafting of the article; SD: Imaging, critical revision of the article; PK: imaging; AW/TM/JWM: critical revision of article; DK: Drafting and revision of the article.

Conflict of interest: none

\section{References}


1. Kalbacher D, Schafer U, RS VB, et al. Long-term outcome, survival and predictors of mortality after MitraClip therapy: Results from the German Transcatheter Mitral Valve Interventions (TRAMI) registry. Int J Cardiol. 2019;277:35-41.

2. Lurz P, Stephan von Bardeleben R, Weber M, et al. Transcatheter Edge-to-Edge Repair for Treatment of Tricuspid Regurgitation. J Am Coll Cardiol. 2021;77(3):229-239.

3. Meggiolaro M, Scatto A, Zorzi A, et al. Confirmation of correct central venous catheter position in the preoperative setting by echocardiographic "bubble-test". Minerva Anestesiol.2015;81(9):989-1000.

4. E. Z. Ueber den Bau und die Entstehung der endocaridtischen Efflorescenzen. Ver Kong Inn Med. 1888;7: $339-343$.

5. el-Shami K, Griffiths E, Streiff M. Nonbacterial thrombotic endocarditis in cancer patients: pathogenesis, diagnosis, and treatment. Oncologist. 2007;12(5):518-523.

6. Deppisch LM, Fayemi AO. Non-bacterial thrombotic endocarditis: clinicopathologic correlations. Am Heart J. 1976;92(6):723-729.

7. Gonzalez Quintela A, Candela MJ, Vidal C, Roman J, Aramburo P. Non-bacterial thrombotic endocarditis in cancer patients. Acta Cardiol. 1991;46(1):1-9.

8. Kuramoto K, Matsushita S, Yamanouchi H. Nonbacterial thrombotic endocarditis as a cause of cerebral and myocardial infarction. Jpn Circ J. 1984;48(9):1000-1006.

9. Lopez JA, Ross RS, Fishbein MC, Siegel RJ. Nonbacterial thrombotic endocarditis: a review. Am Heart J. 1987;113(3):773-784.

\section{Hosted file}

Figures Case.ppt available at https://authorea.com/users/399154/articles/511705-bubblingthe-diagnosis-incidental-finding-of-non-bacterial-thrombotic-endocarditis-in-a-patientwith-pancreatic-carcinoma 